Pacific Journal of Mathematics

COMPLETELY REGULAR ABSOLUTES AND PROJECTIV 


\title{
COMPLETELY REGULAR ABSOLUTES AND PROJECTIVE OBJECTS
}

\author{
R. F. Dickman, JR., J. R. Porter, and L. R. Rubin
}

The absolute $\left(E X, \pi_{X}\right)$ is constructed for an arbitrary space $X$ and is shown to be unique with respect to $E X$ being extremally disconnected and completely regular and $\pi_{X}$ being a $\theta$-continuous, perfect, separating irreducible surjection. A function $f: X \rightarrow Y$ is said to have a continuous $E$-lifting if there is a continuous function $F: E X \rightarrow E Y$ such that $\pi_{Y} \circ F=f \circ \pi_{X}$. A class of functions, called $\eta$-continuous, is introduced, shown to contain the class of continuous functions and the class of $\theta$-continuous, closed surjections, and proved to have continuous $E$-liftings. Functions which have continuous $E$-liftings are completely characterized as being the composition of $\eta$-continuous functions.

1. Introduction and preliminaries. In 1963, Iliadis (see [7]) constructed, for a Hausdorff space $X$, an extremally disconnected Tychonoff space $E X$ and an irreducible, perfect $\theta$-continuous surjection $\pi_{X}: E X \rightarrow X$ and showed that $\left(E X, \pi_{X}\right)$ is unique in this sense: If $Y$ is an extremally disconnected, Tychonoff space and $f: Y \rightarrow X$ is an irreducible, perfect, $\theta$-continuous surjection, then there is a homeomorphism $g: E X \rightarrow Y$ such that $f \circ g=\pi_{X}$. In 1969, Mioduszewski and Rudolf [9] modified this construction to obtain a space $a X$ which has the same underlying set as $E X$ and the topology of $a X$ is generated by the topology of $E X$ plus $\left\{\pi_{X}^{-1}(U): U\right.$ open in $\left.X\right\}$. The function $a_{X}: a X \rightarrow X$ is the same as the function $\pi_{X}$. The space $a X$ is extremally disconnected and Hausdorff, and the function $a_{X}$ is an irreducible, perfect continuous surjection. Also, $\left(a X, a_{X}\right)$ is shown to be unique in the sense similar to the uniqueness of $\left(E X, \pi_{X}\right)$. So, there is a trade-off - the Tychonoffness of $E X$ is reduced to Hausdorff for $a X$, but the $\theta$-continuity of $\pi_{X}$ is strengthened to continuity for $a_{X}$. Both $E X$ and $a X$ are called absolutes of $X$.

More recently, Sapiro [11] and Ul'janov [13] extended the construction and uniqueness of $\left(a X, a_{X}\right)$ ( $a X$ is denoted in [11] by $\left.q X\right)$ for an arbitrary topological space $X$. In this case, $a X$ is extremally disconnected and $a_{X}$ is a separating, irreducible perfect continuous surjection. Also, they showed if $f: X \rightarrow Y$ is a continuous function between spaces $X$ and $Y$, there is a continuous function $F: a X \rightarrow$ $a Y$ such that $a_{Y} \circ F=f \circ a_{X}$.

In the second section of this paper we characterize the projective objects in the category of spaces and perfect separating continuous functions as morphisms. As a cosequence, we obtain the 
result by Sapiro and Ul'janov [11, 13] that continuous functions can be lifted to a continuous function between their $a$-absolutes. For an arbitrary space $X,\left(E X, \pi_{X}\right)$ is constructed and proved to be unique in the fourth section.

In the third section, we introduce the class of $\eta$-continuous functions and show that this class contains several large and interesting classes. In the final section, a necessary and sufficient condition for a function $f: X \rightarrow Y$ to be lifted to a continuous function between their $E$-absolutes is developed in terms of a homomorphism between the Boolean algebras of regular open sets of $Y$ and $X$. It is shown that an $\eta$-continuous function can be lifted to a continuous function between their $E$-absolutes. A function between spaces is shown to be liftable between $E$-absolutes if and only if it is the composition of $\eta$-continuous functions. An example is given of a non- $\eta$-continuous function which is the composition of $\eta$-continuous functions.

In the remainder of this section some necessary definitions and preliminary results are presented. The concept "completely regular" does not necessarily include Hausdorff whereas "Tychonoff" means "completely regular and Hausdorff." A space is said to be extremally disconnected if disjoint open sets have disjoint closures; thus, an extremally disconnected space is not necessarily Hausdorff.

Let $f: X \rightarrow Y$ be a function between two spaces $X$ and $Y$. The function $f$ is irreducible if $f$ is onto and for each closed set $A \subseteq X$ and $A \neq X, f(A) \neq Y$, is perfect if $f$ is closed and compact (i.e., $f^{-1}(y)$ is compact for each $y \in Y$ ), is $\theta$-continuous (respectively, weakly continuous) if for each $x \in X$ and open neighborhood $U$ of $f(x)$, there is an open neighborhood $V$ of $x$ such that $f(\operatorname{cl} V) \cong \operatorname{cl} U$ (respectively, $f(V) \subseteq \mathrm{cl} U$ ) and is separating if whenever $f(x)=f(y)$ and $x \neq y$, then $x$ and $y$ are contained in disjoint neighborhoods of $X$. Clearly a continuous function is $\theta$-continuous, a $\theta$-continuous function is weakly continuous, and a $\theta$-continuous function into a regular space is continuous. Also, composition of $\theta$-continuous (resp. continuous separating) functions is $\theta$-continuous (resp. continuous separating). If $A \subseteq X$, then $f \sharp(A)$ is used to denote $\left\{y \in Y: f^{-1}(y) \subseteq A\right\}$. Note that $f \#(A)=Y \backslash f(X \backslash A)$. Thus, if $A$ is open and $f$ is closed, then $f \#(A)$ is open.

Proposition 1.1. Let $f: X \rightarrow Y$ be an onto function where $X$ and $Y$ are spaces.

(a) Then $f$ is irreducible if and only if for every nonempty open set $U \subseteq X, f \sharp(U) \neq \varnothing$.

(b) If $f$ is closed and $U \subseteq X$ is open, then int $f(U) \supseteqq f \sharp(U)$.

(c) If $f$ is irreducible, closed and weakly continuous and $U \subseteq X$ is open, then $f(U) \subseteq \operatorname{cl} f \#(U)$. 
(d) [12] If $f$ is compact, there is a closed subspace $S \subseteq X$ such that $\left.f\right|_{s}: S \rightarrow Y$ is irreducible.

(e) [7] If $f$ is irreducible, closed, $\theta$-continuous and separating and $Y$ is extremally disconnected, then $f$ is one-to-one; in particular, if $f$ is also continuous, then $f$ is a homeomorphism.

Proof. The proof of (a), (b), and (c) are straightforward.

Let $X, Y, Z$ be spaces and $f: X \rightarrow Y$ and $g: Z \rightarrow Y$ be functions. Let $P=\{(x, z) \in X \times Z: f(x)=g(z)\}, p_{Z}: P \rightarrow Z:(x, z) \rightarrow z$ and $p_{X}: P \rightarrow$ $X:(x, z) \rightarrow x$. Then $\left(P, p_{Z}, p_{X}\right)$ is the pullback of $f: X \rightarrow Y$ and $g: Z \rightarrow Y$, and the pullback square is the following commutative diagram (see [5]):

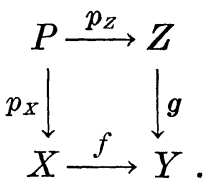

Note that $p_{X}$ and $p_{Z}$ are continuous.

Proposition 1.2. Let $\left(P, p_{Z}, p_{X}\right)$ be the pullback of $f: X \rightarrow Y$ and $g: Z \rightarrow Y$ where $X, Y, Z$, are spaces.

(a) If $f$ is onto, then so is $p_{Z}$.

(b) If $f$ is compact, then so is $p_{Z}$.

(c) If $f$ is separating, then so is $p_{Z}$.

(d) If $f$ is perfect and $g$ is continuous, then $p_{Z}$ is closed.

Proof. The proof is left to the reader.

The category of all topological spaces with separating continuous functions as morphisms is denoted by $\mathrm{TOP}_{s}$; the subcategory of all spaces with perfect separating continuous functions as morphisms is denoted as $\mathrm{TOP}_{s p}$. If $\mathscr{A}$ is a class of morphisms of a category $\mathscr{C}$, then an object $P$ in $\mathscr{C}$ is called $\mathscr{A}$-projective if for $X, Y \in \mathrm{ob}(\mathscr{C})$, and $f \in \operatorname{Mor}(X, Y) \cap \mathscr{A}$, and $g \in \operatorname{Mor}(P, Y)$, there is a $h \in \operatorname{Mor}(P, X)$ such that $f \circ h=g$, i.e., this diagram commutes:

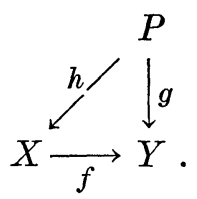

If $\mathscr{A}$ is the class of onto functions, then $\mathscr{A}$-projective objects are called projective, and $\mathscr{P}$ is used to denote the class of perfect, onto functions. An excellent survey about $\mathscr{P}$-projective objects in 
categories of topological spaces has recently been written by Woods [15].

Let $X$ be a space and $L X$ the set of all open ultrafilters on $X$

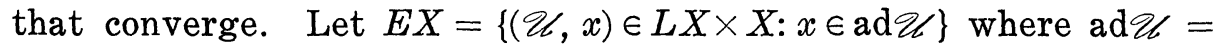
$\cap\{\mathrm{cl} U: U \in \mathscr{Q}\}$, and for each open set $U$ of $X$, let $o U=\{(\mathscr{U}, x) \in$ $E X: U \in \mathscr{Q}\}$. For open sets $U$ and $V$ in $X$, we have $o U \cap o V=o(U \cap V)$, $o U \cup o V=o(U \cup V), o(\phi)=\phi, o X=E X, \quad E X \backslash o U=o(X \backslash \mathrm{cl} U)$, and $o U=o(\operatorname{int}(\operatorname{cl} U))$. Thus, $\{o U: U$ open in $X\}$ forms an open basis for a topology on $E X$. Define the function $\pi_{X}: E X \rightarrow X$ by $\pi_{X}(\mathscr{L}, x)=x$. Also, $\left\{0 U \cap \pi_{X}^{-1}(V): U, V\right.$ open in $\left.X\right\}$ is closed under finite intersection and is an open basis for a topology on $E X ; E X$ with this topology is denoted as $a X$. The function $a X \rightarrow X: p \rightarrow \pi_{X}(p)$ is denoted as $a_{X}$. If $X$ is Hausdorff, then $E X$ and $a X$ can be identified as spaces with $L X$ as the underlying set. So, $a X$ and $E X$ extend the construction of Iliadis [7] and Mioduszewski and Rudolf [9]. Note that for open $U$ in $X, \pi_{X}(o U)=\mathrm{cl}_{X} U=a_{X}(o U)$.

Proposition 1.3. Let $X$ be a space.

(a) [11] $a X$ is extremally disconnected and $a_{X}: a X \rightarrow X$ is a separating, perfect, irreducible continuous surjection.

(b) EX is extremally disconnected and completely regular and $\pi_{X}: E X \rightarrow X$ is a separating, perfect, irreducible $\theta$-continuous surjection.

Proof. The proof of (b) parallels the proof of part (a) (see [11]).

An open set $U$ in a space $X$ is called regular-open if $U=$ int $(\mathrm{cl} U)$. The set of all regular-open sets is a basis for a topology on the underlying set of $X ; X_{s}$ is used to denote this new space. A space $X$ is said to be semiregular if $X=X_{s}$; in particular, it follows that $X_{s}$ is semiregular. If $A \subseteq X$, then int $_{s} A$ (resp. $\left.\operatorname{cl}_{s} A\right)$ is used to denote the interior (resp. closure) of $A$ in $X_{s}$.

Proposition 1.4.

(a) For a space $X,(a X)_{s}=E X$.

(b) An extremally disconnected, semiregular space is completely regular (not necessarily $T_{1}$ ).

(c) A space $X$ is extremally disconnected if and only if $X_{s}$ is extremally disconnected.

Proof. Part (a) follows from this easily proven result: If $U$ and $V$ are open sets in $X$, then $a_{X}^{-1}(U \cap V) \subseteq o U \cap a_{X}^{-1}(V) \subseteq o(U \cap V)$ by Proposition 1 of [11], and $\mathrm{cl}_{a X} a_{X}^{-1}(U)=o U$. Part (b) follows since 
a regular-open set in an extremally disconnected space is cl open. The proof of (c) is easy.

Thus, for a space $X$, the identity function $s_{X}: a X \rightarrow E X$ is a continuous bijection and $s_{X}^{-1}$ is $\theta$-continuous. So, a function $F: a X \rightarrow$ $a Y$ induces the same function $G: E X \rightarrow E Y$ such that $s_{Y} \circ F=G \circ s_{X}$ and conversely, a function $G: E X \rightarrow E Y$ induces a function $F: a X \rightarrow$ $a Y$ such that $s_{Y} \circ F=G \circ s_{X}$. If $f: X \rightarrow Y$ is a function, then $f \circ a_{X}=$ $a_{Y} \circ F$ if and only if $\pi_{Y} \circ G=f \circ \pi_{X}$. If $f \circ a_{X}=a_{Y} \circ F, F$ is called an a-lifting of $f$, and if $f \circ \pi_{Y}=\pi_{Y} \circ G, G$ is called an E-lifting of $f$.

Proposition 1.5. Let $X$ and $Y$ be spaces and $F: a X \rightarrow a Y$ and $G: E X \rightarrow E Y$ be functions such that $s_{Y} \circ F=G \circ s_{X}$. Then

(a) $F$ is $\theta$-continuous if and only if $G$ is continuous.

(b) If $f: X \rightarrow Y$ is continuous, and $G$ is a continuous E-lifting of $f$, then $F$ is also continuous.

Proof. If $G$ is continuous, then $F=s_{Y}^{-1} \circ G \circ s_{X}$ is the composition of $\theta$-continuous functions and, hence is $\theta$-continuous. If $F$ is $\theta$ continuous, then $G=s_{Y} \circ F \circ s_{X}^{-1}$ is the composition of $\theta$-continuous functions and so is $\theta$-continuous. Since $E Y$ is completely regular, then $G$ is continuous. To prove (b), suppose $f$ and $G$ are continuous and $U$ and $V$ are open sets in $Y$. Now, $o U \cap a_{Y}^{-1}(V)$ is basic open set in $a Y$ and $F^{-1}\left(o U \cap a_{Y}^{-1}(V)\right)=F^{-1}(o U) \cap F^{-1}\left(a_{Y}^{-1}(V)\right)=G^{-1}(o U) \cap$ $a_{Y}^{-1}\left(f^{-1}(V)\right)$ is open. Hence, $F$ is continuous.

2. Projective objects. In this section, we show that the projective objects in $\mathrm{TOP}_{s p}$ and the $\mathscr{P}$-projective objects in $\mathrm{TOP}_{s}$ are precisely the extremally disconnected spaces and use this result to obtain Sapiro's results [11] about $a$-liftings. First, a preliminary result is needed.

Proposition 2.1. Suppose $f: X \rightarrow Y$ is a perfect separating surjection, $E$ is extremally disconnected and $g: E \rightarrow Y$ is a continuous function. There is a continuous function $h: E \rightarrow X$ such that $f \circ h=g$.

(a) If, in addition, $g$ is separating, then $h$ is separating.

(b) If, in addition, $f$ is continuous and $g$ is perfect, then $h$ is perfect.

Proof. Let $\left(P, p_{E}, p_{X}\right)$ be the pullback of $f: X \rightarrow Y$ and $g: E \rightarrow Y$. By $1.2, p_{E}$ is a perfect, separating, continuous surjection and $p_{X}$ is continuous. By 1.1, there is a closed set $C \cong P$ such that $p_{E} \mid C$ is irreducible. Let $q_{E}=p_{E} \mid C$ and $q_{X}=p_{X} \mid C$. Since $C$ is closed, $q_{E}$ is 
perfect, separating and continuous. By 1.1, $q_{E}$ is a homeomorphism. Thus, $h=q_{X} \circ\left(q_{E}\right)^{-1}$ is continuous and $f \circ h=g$. If, in addition, $g$ is separating, then by 1.2, $p_{X}$ is separating. So, $q_{X}$ and $h$ are separating. If $f$ is continuous and $g$ is perfect, then by $1.2, p_{X}$ is perfect. Since $C$ is closed, then $q_{X}$ is perfect. So, $h$ is perfect.

The conclusion of Proposition 2.1 is very interesting. Even though a continuous function $h$ is obtained in 2.1, continuity of $f$ is not required in the hypothesis.

THEOREM 2.2. The projective objects of $\mathrm{TOP}_{s p}$ are precisely the extremally disconnected spaces.

Proof. Let $X$ be a projective object in $\mathrm{TOP}_{s p}$. Let $U$ be an openset in $X, V=X \backslash d V, Y$ the topological sum of $\operatorname{cl} U$ and $\operatorname{cl} V$, and $f: Y \rightarrow X: t \rightarrow t$. Now, $f$ is perfect, separating, continuous and onto. Also, the identity function $1_{X}: X \rightarrow X$ is a perfect, separating continuous function. So, there is a perfect, separating continuous function $h: X \rightarrow Y$ such that $f \circ h=1_{X}$. It easily follows that $h(x)=x$ for $x \in U \cup V, h\left(\operatorname{cl}_{X} U\right) \subseteq \operatorname{cl}_{X} U$, and $h\left(\operatorname{cl}_{X} V\right) \subseteq \mathrm{cl}_{X} V$. Since $\operatorname{cl}_{X} U \cap$ $\operatorname{cl}_{X} V=\varnothing$ in $Y$, then it follows that $\operatorname{cl}_{X} U \cap \operatorname{cl}_{X} V=\varnothing$ in $X$. Thus, $X$ is extremally disconnected. Conversely, suppose $E$ is an extremally disconnected space. Let $f: X \rightarrow Y$ be a perfect separating continuous onto function and $g: E \rightarrow Y$ be a perfect separating continuous function. By 2.1, there is a perfect separating continuous function $h: E \rightarrow X$ such that $f \circ h=g$.

CoROLLARY 2.3. The $\mathscr{P}$-projective objects of $\mathrm{TOP}_{s}$ are precisely the extremally disconnected spaces.

Proof. The proof of one part of this corollary uses the same proof as in the first part of 2.2. The other part of this corollary follows from 2.1 .

The sufficiency part of the next result was established by Ul'janov [13] and Sapiro [11] by entirely different methods; they failed to record the reverse implication.

Proposition 2.4. [11] If $X$ and $Y$ are spaces and $\varphi: X \rightarrow Y$ is a function, then $\varphi$ has a continuous a-lifting if and only if $\varphi$ is continuous.

Proof. Suppose $\varphi$ is continuous. Let $f=a_{Y}$ and $g=\varphi \circ a_{X}$. By 1.3, $f$ is a perfect separating continuous surjection. Since $g$ is con- 
tinuous, then by 2.1 , there is a continuous function $h: a X \rightarrow a Y$ such that $f \circ h=g$, i.e., $a_{Y} \circ h=\varphi \circ a_{X}$. So, $\varphi$ has a continuous $a$ lifting. Conversely, suppose $h: a X \rightarrow a Y$ is a continuous function such that $a_{Y} \circ h=\varphi \circ a_{X}$. To show $\varphi$ is continuous, let $A$ be a closed set in $Y$. Since $a_{X}$ is onto, then $\varphi^{-1}(A)=a_{X}\left(h^{-1}\left(a_{Y}^{-1}(A)\right)\right)$; since $a_{X}$ is a closed map, $\varphi^{-1}(A)$ is a closed set. Thus, $\varphi$ is continuous.

A function $\varphi: X \rightarrow Y$ is called a $c$-function if $\operatorname{int} \varphi^{-1}(\mathrm{bd} U)=\varnothing$ for each regular-open set $U$. Sapiro [11] showed that a continuous function has unique $a$-lifting if and only if it is a $c$-function. $\mathrm{He}$ proved that being a $c$-function is sufficient for uniqueness in a straightforward manner but needs his multifunction technique to prove it is necessary. We now use the technique developed in the proof of Proposition 2.1 to prove the necessity for the following result.

Proposition 2.5. [11] Let $X$ and $Y$ be spaces. A function $\varphi: X \rightarrow Y$ has a unique a-lifting if and only if $\varphi$ is a continuous c-function.

Proof. Assume $\phi$ is continuous and there is a regular-open set $U \subseteq Y$ such that int $\varphi^{-1}(\mathrm{bd} U) \neq \varnothing$. Since $a_{X}$ is continuous and onto, then int $g^{-1}(\mathrm{bd} U) \neq \varnothing$ where $g=\varnothing \circ a_{X}$. Let $f=a_{Y}$. To show $\varphi$ has distinct $a$-liftings, it suffices by the proof of 2.1 to find distinct closed sets $C$ and $C^{\prime}$ of $P$ such that $p_{a X} \mid C$ and $p_{a X} \mid C^{\prime}$ are irreducible. Thus, it suffices to find closed sets $P_{1}$ and $P_{2}$ of $P$ and some $z \in a Y$ such that $p_{a Y}^{-1}(z) \cap P_{1} \cap P_{2}=\varnothing$ and $p_{a X}\left(P_{1}\right)=p_{a X}\left(P_{2}\right)=a X$. Let $P_{1}=$ $P \backslash\left(o U \times\right.$ int $\left.g^{-1}(\mathrm{bd} U)\right)$, and for $V=Y \backslash \mathrm{cl} U$, let $P_{2}=P \backslash(o V \times$ int $g^{-1}($ bd $\left.U)\right)$. The closed subsets $P_{1}$ and $P_{2}$ of $P$ and any $z \in a Y$ such that $a_{Y}(z) \in g\left(\right.$ int $g^{-1}($ bd $\left.U)\right)$ have the desired properties.

3. $\eta$-continuous functions. In this section we introduce the class of $\eta$-continuous functions and show that several important classes of functions are $\eta$-continuous. At the end of this section, we develop a characterization of $\eta$-continuous functions that will be used in $\S 5$ to show that $\eta$-continuous functions have continuous $E$-liftings.

For a space $X, R O(X)$ is used to denote the set of all regularopen sets of $X$ and for $U \in R O(X), X \backslash \mathrm{cl} U$ is denoted by $U^{c}$. Thus, $R O(X)$ is an open basis for the topology on $X_{s}$.

Proposition 3.1. Let $f: X \rightarrow Y$ be a function and $U$ be open set in $Y$. Then

(a) $\operatorname{cl} \operatorname{int} f^{-1}(b d U) \cap \operatorname{int} \operatorname{cl} f^{-1}(U)=\varnothing$,

(b) int $f^{-1}(\operatorname{cl} U) \backslash \mathrm{cl} \operatorname{int} f^{-1}(b d U) \subseteq \operatorname{int} \operatorname{cl} f^{-1}(U)$, and 
(c) if $f$ is weakly continuous, $f\left(\operatorname{cl} f^{-1}(U)\right) \leqq \mathrm{cl} U$.

Proof.

Ad(a). Since $f^{-1}(\mathrm{bd} U) \cap f^{-1}(U)=\varnothing$, then $\quad$ int $f^{-1}(\mathrm{bd} U) \cap$ cl $f^{-1}(U)=\varnothing$. Thus, cl int $f^{-1}(\mathrm{bd} U) \cap \operatorname{int} \mathrm{cl} f^{-1}(U)=\varnothing$.

$\operatorname{Ad}(\mathrm{b})$. It suffices to show int $f^{-1}(\mathrm{cl} U) \subseteq \mathrm{cl} f^{-1}(U) \cup \operatorname{int} f^{-1}(\mathrm{bd} U)$. Now, int $f^{-1}(\operatorname{cl} U) \backslash \operatorname{cl~} f^{-1}(U) \cong f^{-1}(\operatorname{cl} U) \backslash f^{-1}(U)=f^{-1}($ bd $U)$. Thus, int $f^{-1}(\operatorname{cl} U) \backslash \operatorname{cl} f^{-1}(U) \subseteq \operatorname{int} f^{-1}(\operatorname{bd} U)$ and int $f^{-1}(\operatorname{cl} U) \subseteq \operatorname{cl} f^{-1}(U) \cup$ int $f^{-1}(b d U)$.

$\operatorname{Ad}(\mathrm{c})$. Let $x \in \mathrm{cl} f^{-1}(U)$. If $f(x) \in V=Y \backslash \mathrm{cl} U$, then $x \in$ int $f^{-1}(\operatorname{cl} V) \leqq X \backslash \mathrm{cl} f^{-1}(U)$, a contradiction. So, $f(x) \in \operatorname{cl} U$.

Proposition 3.2. Let $f: X \rightarrow Y$ be a weakly continuous function. The following are equivalent:

(a) $f^{-1}(U) \subseteq \operatorname{int} \operatorname{cl~} f^{-1}(U)$ for every $U \in R O(Y)$,

(b) $f\left(\mathrm{cl}\right.$ int $\left.f^{-1}(\mathrm{bd} U)\right) \leqq \mathrm{bd} U$ for every $U \in R O(Y)$,

(c) $f\left(\mathrm{cl}\right.$ int $\left.f^{-1}(\mathrm{bd} U)\right) \subseteq \mathrm{cl} U$ for every $U \in R O(Y)$, and

(d) $f\left(\operatorname{cl}\right.$ int $\left.f^{-1}(\operatorname{cl} U)\right) \subseteq \operatorname{cl} U$ for every $U \in R O(Y)$.

Proof. To show (a) implies (b), let $x \in \operatorname{cl} \operatorname{int} f^{-1}(\mathrm{bd} U)$ and assume $f(x) \notin$ bd $U$. Then $\quad x \in f^{-1}\left(U \cup U^{c}\right)$. By (a), $\quad x \in \operatorname{int} c f^{-1}(U) \cup$ int cl $f^{-1}\left(U^{c}\right)$. So, $x \in \operatorname{cl}$ int $f^{-1}($ bd $U) \cap\left(\operatorname{int} \operatorname{cl~} f^{-1}(U) \cup \operatorname{int} \operatorname{cl} f^{-1}\left(U^{c}\right)\right.$ ), a contradiction by $3.1(\mathrm{a})$. Hence, $f(x) \in$ bd $U$.

Clearly, (b) implies (c); since bd $U=$ bd $U^{c}=\operatorname{cl} U \cap \mathrm{cl} U^{c}$, then (c) implies (b). To show (b) implies (a), let $U \in R O(Y)$. Since $f$ is weakly continuous, $f^{-1}(U) \subseteq \operatorname{int} f^{-1}(\mathrm{cl} U) \backslash f^{-1}(\mathrm{bd} U)$. By (b) and 3.1(b), $f^{-1}(U) \leqq \operatorname{int} \mathrm{cl} f^{-1}(U)$. Thus, (a), (b), and (c) are equivalent. Clearly, (d) implies (c). It remains to show (b) implies (d). Let $z \in \mathrm{cl}$ int $f^{-1}(\mathrm{cl} U)$. If $z \in \operatorname{cl} f^{-1}(U)$, then by $3.1(\mathrm{c}), f(z) \in \mathrm{cl} U$. So, suppose $z \notin \operatorname{cl~} f^{-1}(U)$. Then $H=\operatorname{int} f^{-1}(\operatorname{cl} U) \backslash \operatorname{cl} f^{-1}(U) \subseteq \operatorname{int} f^{-1}($ bd $U)$ and $z \in \operatorname{cl} H$ as $z \in \operatorname{cl} \operatorname{int} f^{-1}(\operatorname{cl} U)$. So, $f(z) \in f(\operatorname{cl} H) \subseteq f\left(\operatorname{cl} \operatorname{int} f^{-1}(\operatorname{bd} U)\right) \subseteq$ bd $U$, by (b). Thus, $f\left(\operatorname{cl}\right.$ int $\left.f^{-1}(\operatorname{cl} U)\right) \subseteq$ cl $U$ and (d) is true.

Definition. A function $f: X \rightarrow Y$ is $\eta$-continuous if for $U, V \in$ $R O(Y)$,

(i ) $f^{-1}(U) \subseteq \operatorname{int} \operatorname{cl} f^{-1}(U)$.

(ii) int cl $f^{-1}(U \cap V)=\operatorname{int} \operatorname{cl} f^{-1}(U) \cap \operatorname{int} \operatorname{cl} f^{-1}(V)$.

T. Husain called functions satisfying condition (i) almost continuous functions [6]. An $\eta$-continuous function satisfies (b), (c) and (d) of 3.2 if we can show $\eta$-continus functions are weakly continuous. More in shown in the next proposition.

Proposition 3.3. 
(a) A continuous function is $\eta$-continuous.

(b) If $f: X \rightarrow Y$ is a function and $f^{-1}(U)$ is open for all $U \in$ $R O(Y)$, then $f$ is $\eta$-continuous.

(c) An $\eta$-continuous function is $\theta$-continuous and hence weakly continuous.

Proof. Clearly (a) follows from (b). A function satisfying the hypothesis of (b) satisfies (i) of $\eta$-continuity; part (ii) of $\eta$-continuity follows from the well-known result that if $A$ and $B$ are open, then int cl $(A \cap B)=\operatorname{int} \mathrm{cl} A \cap \operatorname{int} \operatorname{cl} B$. To show (c), let $f: X \rightarrow Y$ be $\eta$ continuous, $x \in X$ and $W$ be an open neighborhood of $f(x)$. Let $U=$ int cl $W$ and $V=U^{c}$. Then $\phi=\operatorname{int} \operatorname{cl} f^{-1}(U \cap V)=\operatorname{int} \operatorname{cl}^{-1}(U) \cap$ int cl $f^{-1}(V)$. Since $x \in f^{-1}(W) \subseteq f^{-1}(U) \subseteq \operatorname{int} \mathrm{cl} f^{-1}(U)$, then $x \in P=$ $X \backslash \mathrm{cl}$ int $\mathrm{cl} f^{-1}(V)$. So, cl $P=X \backslash$ int $\mathrm{cl} f^{-1}(V) \subseteq X \backslash f^{-1}(V)$, and $f(\operatorname{cl} P) \subseteq$ $Y \backslash V=\operatorname{cl} U=\operatorname{cl} W$.

So, by 3.3, the class of continuous functions is a large class of $\eta$-continuous functions. The next lemma is used to develop another class of $\eta$-continuous functions.

LEMMA 3.4. Let $f: X \rightarrow Y$ be a weakly continuous function satisfying these two conditions:

(a) For each $U \in R O(Y), f^{-1}(U) \leqq \operatorname{int} \operatorname{cl} f^{-1}(U)$.

(b) For each nonempty open set $W$ in $X$, int $f(W) \neq \phi$. Then for $U, V \in R O(Y)$, int $\operatorname{cl} f^{-1}(U \cap V)=\operatorname{int} \operatorname{cl} f^{-1}(U) \cap$ int $\operatorname{cl} f^{-1}(V)$.

Proof. It is straightforward to show int cl $f^{-1}(U \cap V) \subseteq$ int cl $f^{-1}(U) \cap \operatorname{int} \operatorname{cl} f^{-1}(V)$. Let $R=\left[\operatorname{intcl~} f^{-1}(U) \cap \operatorname{int} \operatorname{cl} f^{-1}(V)\right] \backslash$ cl int cl $f^{-1}(U \cap V)$. It suffices to show that $R=\varnothing$. Since $f(R) \subseteq$ $f\left(\mathrm{cl} f^{-1}(U)\right) \cap f\left(\operatorname{cl} f^{-1}(V)\right)$, then by $3.1(\mathrm{c}), f(R) \subseteq \operatorname{cl} U \cap \mathrm{cl} V$. By (a), $R \cap f^{-1}(U \cap V)=\varnothing$. So, $f(R) \subseteq(\operatorname{cl} U \cap \operatorname{cl} V) \backslash(U \cap V)$. But $(\operatorname{cl} U \cap \operatorname{cl} V) \backslash(U \cap$ $V) \leqq$ bd $U \cup$ bd $V$ which is nowhere dense. So, int $f(R) \leqq \operatorname{int}($ bd $U U$ bd $V)=\varnothing$. By (b), $R=\varnothing$.

Proposition 3.5. Let $f: X \rightarrow Y$ be a weakly continuous, closed, irreducible surjection. Then

(a) $f$ is $\eta$-continuous,

(b) if $U$ is open in $Y$, then int $\operatorname{cl}^{-1}(U)=\operatorname{int} f^{-1}(\operatorname{cl} U)$, and

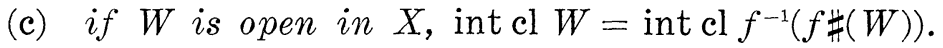

Proof.

$\operatorname{Ad}(a)$. Let $V \in R O(Y)$ and $x \in f^{-1}(V)$. Then there is an open subset $U$ of $X$ containing $x$ with $f(U) \cong \operatorname{cl} V$. Suppose $W$ is a non- 
empty open subset of $U$. Now $f \sharp(W) \neq \varnothing$ and $f \sharp(W) \subseteq \operatorname{cl} V$. So, $f^{\#}(W) \cap V \neq \varnothing$ implying $W \cap f^{-1}(V) \neq \varnothing$. Thus, $f^{-1}(V)$ is dense in $U$, i.e., $U \subseteq \operatorname{cl~} f^{-1}(V)$. Hence, $x \in U \subseteq \operatorname{int~cl~} f^{-1}(V)$. This shows $f^{-1}(V) \subseteq \operatorname{int} \operatorname{cl} f^{-1}(V)$. By 1.1 and 3.4, it follows that $f$ is $\eta$-continuous.

$\operatorname{Ad}(\mathrm{b})$. Let $P=\operatorname{int} \mathrm{cl} f^{-1}(U)$ and $Q=\operatorname{int} f^{-1}(\mathrm{cl} U)$. By 3.1(c), $P \subseteq Q$. To show $Q \subseteq P$, it suffices to show $\operatorname{cl} Q \subseteq \operatorname{cl} P$ since $P$ is regular open. Assume, cl $Q \backslash \operatorname{cl} P \neq \varnothing$, then $Q \backslash \mathrm{cl} P=W$ is a nonempty open set. But by (a), $f^{-1}(U) \subseteq P$; so, $W \cong f^{-1}(\mathrm{cl} U) \backslash f^{-1}(U)=$ $f^{-1}($ bd $U)$ implying $f(W)$ has empty interior. This contradicts 1.1; so, $P=Q$.

$\operatorname{Ad}(\mathrm{c})$. It suffices to show that $\mathrm{cl} W=\operatorname{cl} f^{-1}(f \#(W))$. Clearly, cl $W \supseteqq \operatorname{cl} f^{-1}(f \sharp(W))$. Suppose $\phi \neq T \subseteq W$ where $T$ is open. Then $\phi \neq f \sharp(T)$ and $\phi \neq f^{-1}(f \#(T)) \subseteq T \cap f^{-1}(f \sharp(W))$. Thus, $f^{-1}(f \sharp(W))$ is dense in $W$ implying $W \leqq \operatorname{cl} f^{-1}(f \sharp(W))$. So, $\operatorname{cl} W \leqq \operatorname{cl} f^{-1}(f \sharp(W))$.

Using 3.4 again, we obtain another large class of $\eta$-continuous functions.

Proposition 3.6. A weakly continuous, open function is $\eta$ continuous.

Proof. Suppose $f: X \rightarrow Y$ is open and weakly continuous, and let $U \in R O(Y)$. Clearly, 3.4(b) is satisfied. By weak continuity, $f^{-1}(U) \subseteq \operatorname{int} f^{-1}(\mathrm{cl} U)$. Now, $f\left(\operatorname{int} f^{-1}(\mathrm{bd} U)\right)$ is open and contained in bd $U$. Since $\operatorname{int}($ bd $U)=\varnothing$, then $\operatorname{int} f^{-1}($ bd $U)=\varnothing$. Thus, $f^{-1}(U) \subseteq$ int $f^{-1}(\mathrm{cl} U) \backslash \mathrm{clint} f^{-1}(\mathrm{bd} U)$, and by 3.1(b), $f^{-1}(U) \leqq \operatorname{int} \mathrm{cl} f^{-1}(U)$.

\section{LEMMA 3.7.}

(a) If $U$ is open and $A$ is closed in a space $Y$, then $\operatorname{cl}_{s} U=\mathrm{cl} U$, $\operatorname{int}_{s} A=\operatorname{int} A$, and $\operatorname{int}_{s} \operatorname{cl}_{s} U=\operatorname{int} \mathrm{cl} U$.

(b) For a space $Y, R O(Y)=R O\left(Y_{s}\right)$.

(c) Let $\tau$ and $\sigma$ be topologies on a set $X$. Then $R O(X, \tau)=$ $R O(X, \sigma)$ if and only if the identity functions $i:(X, \tau) \rightarrow(X, \sigma)$ and $i^{-1}:(X, \sigma) \rightarrow(X, \tau)$ are $\eta$-continuous.

(d) If $f: X \rightarrow Y$ is a $\theta$-continuous, closed bijection, then for open $U$ in $Y, f^{-1}\left(\operatorname{int}_{Y} \operatorname{cl}_{Y} U\right)=\operatorname{int}_{X} \mathrm{cl}_{X} f^{-1}(U)$; in particular, for $U \in R O(Y), f^{-1}(U) \in R O(X)$.

Proof. The proof of (b) follows from (a); the proof of (a) is straightforward and left to the reader.

Ad(c). The necessity of the conditions follows from 3.3(b). To prove sufficiency, suppose $i$ and $i^{-1}$ are $\eta$-continuous. By Proposition 1 in [3], $i$ is a homeomorphism between $\left(X, \tau_{s}\right)$ and $\left(X, \sigma_{s}\right)$. So, 
$R O\left(X, \tau_{s}\right)=R O\left(X, \sigma_{s}\right) . \quad$ By (b), it follows that $R O(X, \tau)=R O(X, \sigma)$.

$\operatorname{Ad}(\mathrm{d})$. Let $\tau$ be the topology on $X$. We can assume $Y=X$ with the topology $\sigma$ and $f=i$ is the identity function on $X$. Since $f$ is closed, then $\tau \subseteq \sigma$. By (c), $\tau_{s}=\sigma_{s}$. Since $\sigma_{s} \leqq \tau \leqq \sigma$, then by (a) for $U \in \sigma$, it follows that int $\operatorname{cl}_{\sigma} U=\operatorname{int}_{\tau} \operatorname{cl}_{\tau} U=\operatorname{int}_{s} \operatorname{cl}_{s} U$.

Let $f: X \rightarrow Y$ be a function. Now, $\left\{W \cap f^{-1}(U): W\right.$ open in $X$, $U \in R O(Y)\}$ is closed under finite intersection and forms a basis for a topology on the underlying set of $X$. Let $X^{*}$ denote $X$ with this new topology and $f^{*}: X^{*} \rightarrow Y$ be defined by $f^{*}(x)=f(x)$. Note that the identity $X^{*} \rightarrow X$ is continuous. Now $f^{*}$ is $\eta$-continuous because it satisfies the hypothesis $3.3(\mathrm{~b})$. For $A \subseteq X$, let $\mathrm{cl}^{*} A$ (resp. int* $A$ ) denote the closure of $A$ in $X^{*}$ (resp. interior of $A$ in $X^{*}$ ).

THEOREM 3.8. Let $f: X \rightarrow Y$ be weakly continuous. The following are equivalent:

(a) $f$ is $\eta$-continuous,

(b) the identity function $h: X \rightarrow X^{*}$ is weakly continuous,

(c) $\left(X^{*}\right)_{s}=X_{s}$, and

(d) $R O\left(X^{*}\right)=R O(X)$.

Proof. The equivalence of (c) and (d) follows from 3.7(b). By 3.7(c), (d) implies (b). Suppose $h$ is weakly continuous. Since $h^{-1}: X^{*} \rightarrow X$ is continuous, then $h$ is also closed and irreducible. By $3.5, h$ is $\eta$-continuous, and by $3.7, R O(X)=R O\left(X^{*}\right)$. So, (b) and (d) are equivalent. Suppose (c) is true. Since topology of $X \cong$ topology of $X^{*}$ and $\left(X^{*}\right)_{s}=X_{s}$, then by 3.7(a), it follows that for any open set $W$ in $X^{*}, \mathrm{cl}^{*} W=\operatorname{cl} W=\operatorname{cl}_{s} W$ and hence int* $\mathrm{cl}^{*} W=\operatorname{int} \mathrm{cl} W=$ int $\operatorname{cl}_{s} W$. If $U \in R O(Y)$, then $\left(f^{*}\right)^{-1}(U)$ is open in $X^{*}$ and $f^{-1}(U)=$ $\left(f^{*}\right)^{-1}(U) \leqq \operatorname{int}^{*} \mathrm{cl}^{*}\left(f^{*}\right)^{-1}(U)=\operatorname{int} \mathrm{cl}^{-1}(U)$. Also, if $U, V \in R O(Y)$, then int $\mathrm{cl}^{-1}(U \cap V)=$ int* $^{*} \mathrm{cl}^{*}\left(f^{*}\right)^{-1}(U \cap V)=$ int* $\mathrm{cl}^{*}\left(f^{*}\right)^{-1}(U) \cap$ int* cl* $^{*}\left(f^{*}\right)^{-1}(V)=\operatorname{int} \operatorname{cl} f^{-1}(U) \cap \operatorname{int} \mathrm{cl}^{-1}(V)$. Thus, (c) implies (a). To show (a) implies (b), suppose $f$ is $\eta$-continuous. Let $x \in X$ and $W \cap f^{-1}(V)$ be a basic open subset of $X^{*}$ containing $h(x)=x$. Since $f$ is $\eta$-continuous, we may assume that $W \cong \operatorname{int} c l f^{-1}(V)$. We will show that $h(W) \subseteq \mathrm{cl}^{*}\left(W \cap f^{-1}(V)\right)$. Let $z \in W$ and $S \cap f^{-1}(R)$ be a basic open subset of $X^{*}$ containing $h(z)=z$; again, we may assume that $S \subseteq$ int cl $f^{-1}(R)$. We will show that $S \cap f^{-1}(R) \cap W \cap f^{-1}(V) \neq \varnothing$. Now, $z \in S \cap W \subseteq \operatorname{int} \operatorname{cl} f^{-1}(R) \cap \operatorname{int} \operatorname{cl} f^{-1}(V)=\operatorname{int} \operatorname{cl} f^{-1}(R \cap V)$. Thus, $\phi \neq S \cap W \cap f^{-1}(R \cap V)=S \cap W \cap f^{-1}(R) \cap f^{-1}(W)$. This completes that the proof (a) implies (b).

CoROLlary 3.9. A function $f$ is $\eta$-continuous if and only if $f$ is a composition $s \circ g \circ h$ where $g$ is a continuous function and both $s$ and $h$ are $\theta$-continuous, closed, bijections. 
Proof. Let $f: X \rightarrow Y$ be $\eta$-continuous. As noted in the proof of $3.8, h: X \rightarrow X^{*}$ is weakly continuous, closed and irreducible. By 3.5 and $3.3(\mathrm{c}), h$ is $\theta$-continuous. Now, $f=f^{*} \circ h$. Let $g: X^{*} \rightarrow Y_{s}$ be defined by $g(x)=f^{*}(x)$ for $x \in X^{*}$, and let $s: Y_{s} \rightarrow Y$ be identity function. By the definition of $f^{*}$, it follows that $g$ is continuous. By 3.7(a), $s$ is $\eta$-continuous; since $s^{-1}$ is continuous, then $s$ is an $\eta$ continuous, closed irreducible surjection. So $s$ is $\theta$-continuous by 3.3(c). Now, $f^{*}=s \circ g$. Hence, $f=s \circ g \circ h$ where $g$ is continuous and $s$ and $h$ are $\theta$-continuous, closed, bijections. Conversely suppose $f=s \circ g \circ h: X \rightarrow Y$ where $g: R \rightarrow T$ is continuous and both $s: T \rightarrow Y$ and $h: X \rightarrow R$ are $\theta$-continuous, closed bijections. Let $U, V \in R O(Y)$. By 3.7(d), $s^{-1}(U)$ is open in $T$. Since $g^{-1}\left(s^{-1}(U)\right)$ is open, then int $_{R} \mathrm{cl}_{R} g^{-1}\left(s^{-1}(U)\right) \supseteqq g^{-1}\left(s^{-1}(U)\right)$; it follows that $\operatorname{int}_{X} \mathrm{cl}_{X} f^{-1}(U)=$ $h^{-1}\left(\operatorname{int}_{R} \operatorname{cl}_{R} g^{-1}\left(s^{-1}(U)\right)\right) \supseteqq h^{-1}\left(g^{-1}\left(s^{-1}(U)\right)\right)=f^{-1}(U)$. Since $g^{-1}\left(s^{-1}(U \cap V)\right)$ is open, then $\operatorname{int}_{R} \mathrm{cl}_{R} g^{-1}\left(s^{-1}(U \cap V)\right)=\operatorname{int}_{R} \mathrm{cl}_{R} g^{-1}(U) \cap \operatorname{int}_{R} \mathrm{cl}_{R} g^{-1}\left(s^{-1}(V)\right)$. Thus, int $\operatorname{cl}_{X} f^{-1}(U \cap V)=h^{-1}\left(\operatorname{int}_{R} \operatorname{cl}_{R} g^{-1}\left(s^{-1}(U)\right)\right) \cap h^{-1}\left(\operatorname{int}_{R} \operatorname{cl}_{R} g^{-1}\left(s^{-1}\right.\right.$ $(V)))=\operatorname{int}_{X} \operatorname{cl}_{X} f^{-1}(U) \cap \operatorname{int}_{X} \operatorname{cl}_{X} f^{-1}(V)$. So, $f$ is $\eta$-continuous.

4. Uniqueness of $\left(E X, \pi_{X}\right)$. In this section, we prove that the absolute $\left(E X, \pi_{X}\right)$ as constructed in $\S 1$ for an arbitrary space $X$ is unique with respect to $E X$ being extremally disconnected, completely regular and $\pi_{X}$ being perfect, irreducible, separating and $\theta$-continuous.

A major advantage of using the absolute $E X$ as opposed to $a X$ is that when $X$ is Hausdorff, $E X$ is Tychonoff (see [7]) and has Hausdorff compactifications. In particular, the Stone-Cech compactification $\beta E X$ is the Stone space of the Boolean algebra of regular open sets on $X$; thus, a natural link between a Hausdorff space $X$ and the Stone-Cech compactification of the extremally disconnected space $E X$ exists. In [2], this link is utilized to give a characterization of Martin's Axiom in the class of $H$-closed spaces.

For a function $f: Y \rightarrow X, Y^{\prime}$ is used to denote the underlying set of $Y$ plus the topology generated by the basis $\left\{f^{-1}(V) \cap U: V\right.$ open in $X$ and $U$ open in $Y$. Define $f^{\prime}: Y^{\prime} \rightarrow X$ by $f^{\prime}(y)=f(y)$ for all $y \in Y^{\prime}$. Clearly, $f^{\prime}$ is continuous. For $A \subseteq Y^{\prime}$, int' $A\left(\right.$ resp. cl $\left.^{\prime} A\right)$ is used to denote the interior (resp. closure) of $A$ in $Y^{\prime}$. Note that $Y^{\prime}$ is slightly different from $Y^{*}$ as developed in $\S 3$ for 3.7 .

Proposition 4.1. Let $f: Y \rightarrow X$ be a closed irreducible, $\theta$-continuous surjection.

(a) If $W$ is open in $Y$ and $V$ is open in $X$, then int' $\operatorname{cl}^{\prime}(W \cap$ $\left.f^{-1}(V)\right)=\operatorname{int} \operatorname{cl}\left(W \cap f^{-1}(V)\right)$.

(b) $\left(Y^{\prime}\right)_{s}=Y_{s}$.

(c) If $f$ is compact and separating, then $f^{\prime}$ is a perfect irreducible separating continuous surjection. 
Proof. Part (b) follows from (a), and part (c) is straightforward (even through many details must be checked). To show (a), first note that by using the proofs of $3.5(\mathrm{a})$ and $3.4(\mathrm{~b})$, we can show that $f$ is slightly stronger than $\eta$-continuous, i.e., for arbitrary open sets $U, V$ in $X, f$ satisfies:

(i) $f^{-1}(U) \cong \operatorname{int} \mathrm{cl} f^{-1}(U)$ and

(ii) int cl $f^{-1}(U \cap V)=\operatorname{int} \operatorname{cl} f^{-1}(U) \cap \operatorname{int} \operatorname{cl} f^{-1}(V)$.

Now, we will show for an open set $W$ in $Y$ and open set $V$ in $X$, $\operatorname{cl}^{\prime}\left(W \cap f^{-1}(V)\right)=\operatorname{cl}\left(W \cap f^{-1}(V)\right)$. Clearly $\operatorname{cl}^{\prime}\left(W \cap f^{-1}(V)\right) \leqq \operatorname{cl}(W \cap$ $\left.f^{-1}(V)\right)$. Let $y \in \operatorname{cl}\left(W \cap f^{-1}(V)\right)$ and $y \in T \cap f^{-1}(R)$ where $T$ is open in $Y$ and $R$ is open in $X$. Since $f^{-1}(R) \subseteq \operatorname{int} \operatorname{cl} f^{-1}(R)$, then $T \cap$ int cl $f^{-1}(R) \cap W \cap f^{-1}(V) \neq \varnothing$. Assume $f \sharp(T) \cap R \cap f \sharp(W) \cap V=\varnothing$. Then by (ii), int cl $f^{-1}(f \sharp(T)) \cap \operatorname{int} \operatorname{cl} f^{-1}(R) \cap \operatorname{int} \operatorname{cl} f^{-1}(f \sharp(W)) \cap$ int cl $f^{-1}(V)=\varnothing$, and by $3.5(\mathrm{c})$, int cl $T \cap \operatorname{int} \mathrm{cl} f^{-1}(R) \cap \operatorname{int} \mathrm{cl} W \cap$ int cl $f^{-1}(V)=\varnothing$. This latter equality contradicts the fact that $T \cap$ int $\operatorname{cl} f^{-1}(R) \cap W \cap f^{-1}(V) \neq \varnothing$. To complete the proof of (a), it suffices to show that $\operatorname{int}^{\prime}\left(\operatorname{cl}\left(W \cap f^{-1}(V)\right)\right) \subseteq \operatorname{int}\left(\operatorname{cl}\left(W \cap f^{-1}(V)\right)\right)$. Suppose $\phi \neq T \cap f^{-1}(R) \subseteq \mathrm{cl}\left(W \cap f^{-1}(V)\right)$ where $T$ is open in $Y$ and $R$ is open in $X$. By (i), $T \cap f^{-1}(R) \subseteq T \cap \operatorname{int} \operatorname{cl} f^{-1}(R) \leqq \operatorname{int} \operatorname{cl}(T \cap$ $\left.f^{-1}(R)\right) \subseteq \operatorname{int} \operatorname{cl}\left(W \cap f^{-1}(V)\right)$. Thus, int' $\left(\operatorname{cl}\left(W \cap f^{-1}(V)\right)\right) \subseteq \operatorname{int} \operatorname{cl}(W \cap$ $\left.f^{-1}(V)\right)$.

THeOREM 4.2. Let $X$ be a space. If $Y$ is extremally disconnected, completely regular and if $f: Y \rightarrow X$ is a perfect irreducible separating $\theta$-continuous surjection, then there is a homeomorphism $h: E X \rightarrow Y$ such that $f \circ h=\pi_{X}$.

Proof. By (4.1), $f^{\prime}: Y^{\prime} \rightarrow X$ is a perfect irreducible separating surjection and $\left(Y^{\prime}\right)_{s}=Y_{s}$. Since $Y$ is completely regular, then $Y_{s}=Y$. By 1.4, $Y^{\prime}$ is extremally disconnected. By 2.1 , there is a perfect separating continuous function $h: a X \rightarrow Y^{\prime}$ such that $f^{\prime}$ 。 $h=a_{X}$. Since $a_{X}$ is irreducible, then it follows that $h$ is onto and irreducible. By 1.1, $h$ is a homeomorphism. Thus, $h:\left(a X_{s}\right) \rightarrow\left(Y^{\prime}\right)_{s}$ is a homeomorphism. But $E X=(a X)_{s}$, and $Y=\left(Y^{\prime}\right)_{s}$. So, $h: E X \rightarrow Y$ is homeomorphism, and since $f^{\prime} \circ h=a_{X}$, it follows that $f \circ h=\pi_{X}$.

REMARK. 1. The Stone-Cech compactification $\beta Y$ can be constructed for a completely regular space $Y$ which is not necessarily Hausdorff. This can be accomplished by using the same construction in 3.8 in [7]. This extension $\beta Y$ will be compact, completely regular, and Hausdorff except for $Y$ (i.e., if $a, b \in Y, a \neq b$, and $a \notin Y$, then $a$ and $b$ are contained in disjoint open sets in $\beta Y$-see [8]). Now, $\beta Y$ has this maximal property: If $b Y$ is a compact, completely regular extension of $Y$, there is a continuous function 
$f: \beta Y \rightarrow b Y$ such that $f(y)$ for $y \in Y$. This maximal property shows that $\beta Y$ is the usual Stone-Cech compactification of $Y$ when $Y$ is Tychonoff. Also, if $b Y$ is Hausdorff except for $Y$, then $f$ is onto. Thus, for a non-Hausdorff space $X, \beta E X$ is compact, completely regular and Hausdorff except for $E X$.

Let $G X$ be used to denote the Stone space of the complete Boolean algebra $R O(X)$ of regular open sets of $X$. Then $E X$ can be enlarged by defining $\theta X=\{(\mathscr{U}, x): \mathscr{U} \in G X$ and $x \in$ ad $\mathscr{K}\} \cup$ $\{(\mathscr{U}, \infty): \mathscr{C} \in G X$ and ad $\mathscr{C}=\varnothing\}$ with the topology generated by $\{o U: U$ open in $X\}$ where $o U=\{(\mathscr{U}, z) \in \theta X: U \in \mathscr{C}\}$. Now $\theta X$ is an extension of $E X$, and $\beta E X$ and $\theta X$ are equivalent extensions of $E X$, i.e., there is a homeomorphism between $\beta E X$ and $\theta X$ which fixes the points of $E X$.

Also, if $Y$ is a completely regular space, there is an equivalence relation on $Y$ and a topology on the induced partition $Y_{t}$ such that $Y_{t}$ is Tychonoff and $Y$ and $Y_{t}$ have the same ring of real-valued continuous functions via the induced quotient function $\sigma: Y \rightarrow Y_{t}$, i.e., if $f$ is a real-valued continuous function on $Y$, then there is a unique real-valued continuous function $g$ on $Y_{t}$ such that $g \circ \sigma=f$ (see 3.9 of [4]). It follows that $(\theta X)_{t}=G X$, and by the previous paragraph, $(\beta E X)_{t}=G X$.

Thus, when $X$ is not Hausdorff, there is a theory of completely regular, compactifications of $E X$ which is similar to the Hausdorff compactifications of $(E X)_{t}$ and there is a natural link between $\beta E X$ and $G X$.

2. We are indebted to the referee for noting that another application of Proposition 4.1 is that the existence and properties of $\left(a X, a_{X}\right)$ follows from the existence and properties of $\left(E X, \pi_{X}\right)$; in particular, in the notation of $4.1, a X=(E X)^{\prime}$. This construction of $a X$ seems more natural even though $a X$ was discovered first.

5. Continuous $E$-liftings, In this section, we give a necessary and sufficient condition for a function $f: X \rightarrow Y$ to have a continuous $E$-lifting in terms of a Boolean algebra homomorphism $\psi: R O(Y) \rightarrow$ $R O(X)$.

We show that every $\eta$-continuous function has a continuous $E$ lifting and that a function has a continuous $E$-lifting if and only if it is a composition of $\eta$-continuous functions. Also, we give an example of $\eta$-continuous surjections whose composition is not $\eta$ continuous. Finally, we prove that a necessary and sufficient condition for an $\eta$-continuous function to have a unique continuous $E$ lifting is being a $c$-function.

For a space $X$, let $R O(X)$ denote the set of all regular-open sets in $X$ and $O(X)$ the set of all open sets in $X$. Let $S(R O(X))$ denote 
the Stone space corresponding the Boolean algebra $R O(X)$ (see [14]). The elements of $S(R O(X))$ are regular-open ultrafilters on $X$; the topology on $S(R O(X))$ is generated by $\{\lambda(U): U \in R O(X)\}$ where $\lambda(U)=\{\mathscr{U} \in S(R O(X)): U \in \mathscr{W}\}$.

Lemma 5.1. Let $X$ and $Y$ be spaces and $f: X \rightarrow Y$ be a function. If there is a continuous function $F: S(R O(X)) \rightarrow S(R O(Y))$ such that for $\mathscr{C} \in S(R O(X))$ and $x \in \operatorname{ad} \mathscr{U}, f(x) \in \operatorname{ad} F(\mathscr{C})$, then $f$ has a continuous E-lifting.

Proof. For $\mathscr{U} \in S(R O(X)), \mathscr{U}^{\prime}=\{U \in O(X)$ : int cl $U \in \mathscr{W}\}$ is an open ultrafilter; for each open ultrafilter $\mathscr{W}$ on $X, \mathscr{W} \cap R O(X)$ is a regular-open ultrafilter and $(\mathscr{W} \cap R O(X))^{\prime}=\mathscr{W}$ (see [10]). Define $G: E X \rightarrow E Y$ by $G(\mathscr{W}, x)=\left(F(\mathscr{W} \cap R O(X))^{\prime}, f(x)\right) . \quad G$ is a welldefined function since ad $\mathscr{W}=\operatorname{ad} \mathscr{U}^{\prime}$ for $\mathscr{C} \in S(R O(X))$. It is left to the reader to show that $G$ is continuous and $\pi_{Y} \circ G=f \circ \pi_{X}$.

Lemma 5.2. Let $X$ and $Y$ be spaces. If $\phi \neq S \subseteq R O(Y)$ such that $Y \backslash$ cl $U \in S$ whenever $U \in S$ and $\varphi: S \rightarrow R O(X)$ satisfies:

(1) $U \in S$ implies $\varphi(Y \backslash \operatorname{cl} U)=X \backslash \operatorname{cl} \varphi(U)$ and

(2) for $U_{1}, \cdots, U_{n} \in S, U_{1} \cap \cdots \cap U_{n}=\varnothing$ implies $\varphi\left(U_{1}\right) \cap \cdots \cap$ $\varphi\left(U_{n}\right)=\varnothing$, then $\varnothing$ has a unique Boolean algebra homomorphism extension to the Boolean subalgebra generated by $S$.

\section{Proof. This follows from 2.15 in [1].}

THEOREM 5.3. A function $f: X \rightarrow Y$ between spaces has an $E$ lifting if and only if there is a function $\varphi: R O(Y) \rightarrow O(X)$ such that for $U \in R O(Y)$,

(1) $f(\operatorname{cl} \varphi(U)) \subseteq \operatorname{cl} U$,

(2) $\varphi(U) \cap \varphi(Y \backslash \operatorname{cl} U)=\varnothing$ and $\operatorname{cl}(\varphi(U) \cup \varphi(Y \backslash \operatorname{cl} U))=X$, and

(3) for $\quad U_{1}, \cdots, U_{n} \in R O(Y), \quad U_{1} \cap \cdots \cap U_{n}=\varnothing \quad$ implies $\varphi\left(U_{1}\right) \cap \cdots \cap \varphi\left(U_{n}\right)=\varnothing$.

Proof. Suppose $f$ has a continuous $E$-lifting $G: E X \rightarrow E Y$. Let $U \in R O(Y)$. Then $o U$ is clopen in $E Y$ and there is a unique regularopen set $V$ in $X$ such that $o V=G^{-1}(o U)$. Let $\varphi(U)=V$. Then $\varphi$ satisfies (1), (2), and (3). Conversely, suppose there is a function $\varphi: R O(Y) \rightarrow O(X)$ satisfying (1), (2), and (3). Define $\psi: R O(Y) \rightarrow$ $R O(X)$ by $\psi(U)=\operatorname{int} \operatorname{cl} \varphi(U)$. By (2), it follows that $\psi(Y \backslash \operatorname{cl} U)=$ $X \backslash \operatorname{cl} \psi(U)$. If $U_{1} \cap \cdots \cap U_{n}=\varnothing$, then $\phi=\operatorname{int} \operatorname{cl}\left(\varphi\left(U_{1}\right) \cap \cdots \cap \phi\left(U_{n}\right)\right)=$ int $\operatorname{cl} \varphi\left(U_{1}\right) \cap \cdots \cap \operatorname{int} \operatorname{cl} \varphi\left(U_{n}\right)=\psi\left(U_{1}\right) \cap \cdots \cap \psi\left(U_{n}\right)$. By Lemma 5.2, $\psi$ is a Boolean algebra homomorphism. By Stone's Duality Theorem, there is a continuous function $F: S(R O(X)) \rightarrow S(R O(Y))$ such that for 
$\mathscr{W} \in S(R O(X)),\{\psi(W): W \in F(\mathscr{U})\} \subseteq \mathscr{U}$. Suppose $\mathscr{U} \in S(R O(X))$ and $x \in \operatorname{ad} \mathscr{U}$. For $U \in R O(Y), \operatorname{cl} \psi(U)=\operatorname{cl} \varphi(U) \cong f^{-1}(\operatorname{cl} U)$. So, $x \in$ $\cap\{\operatorname{cl} \varphi(W): W \in F(\mathscr{C})\} \subseteq \cap\left\{f^{-1}(\operatorname{cl} W): W \in F(\mathscr{C})\right\}=f^{-1}(\operatorname{ad} F(\mathscr{C}))$. By Lemma 5.1, $f$ has a continuous $E$-lifting.

Corollary 5.4. Suppose $f: X \rightarrow Y$ is a function. Then $f$ has a continuous E-lifting if and only if there is a Boolean algebra homomorphism \&: $R O(Y) \rightarrow R O(X)$ such that for $U \in R O(Y), f^{-1}(U) \subseteq$ $\psi(U) \leqq \operatorname{cl} \psi(U) \leqq f^{-1}(\operatorname{cl} U)$.

Proof. Let $\varphi: R O(Y) \rightarrow O(X)$ be the function generated in Theorem 5.3. Then as in the proof of 5.3, $\psi: R O(Y) \rightarrow R O(X)$ given by $\psi(U)=\operatorname{int} \operatorname{cl} \varphi(U)$ is a Boolean algebra homomorphism. It then follows that for $U \in R O(Y), f^{-1}(U) \subseteq \psi(U) \subseteq \operatorname{cl} \psi(U) \subseteq f^{-1}(\operatorname{cl} U)$.

The Boolean algebra characterization in 5.4 is used to develop a corresponding characterization for $\eta$-continuous surjections.

THEOREM 5.5. An $\eta$-continuous function has a continuous Elifting.

Proof. Let $f: X \rightarrow Y$ be $\eta$-continuous. Since the composition of functions each with a continuous $E$-lifting has a continuous $E$ lifting, then by 3.9 , we need to check when $f$ is continuous and when $f$ is a $\theta$-continuous, closed, bijection. By 2.4 and 1.5, a continuous function has a continuous $E$-lifting. So, suppose $f$ is a $\theta$ continuous, closed, bijection (so, $f$ is irreducible and perfect). Then $f \circ \pi_{X}: E X \rightarrow Y$ is $\theta$-continuous, perfect, irreducible surjection. So, $\left(f \circ \pi_{X}\right)^{\prime}:(E X)^{\prime} \rightarrow Y$ is continuous. By 2.1 , there is a continuous function $h:(E X)^{\prime} \rightarrow E Y$ such that $\pi_{Y} \circ h=\left(f \circ \pi_{X}\right)^{\prime}$. By 4.1, $\left((E X)^{\prime}\right)_{s}=$ $(E X)_{s}=E X$ since $E X$ is completely regular. Thus, the identity function $j: E X \rightarrow(E X)^{\prime}$ is $\theta$-continuous; hence, $h \circ j: E X \rightarrow E Y$ is $\theta$ continuous. Since $E Y$ is completely regular, then $h \circ j$ is continuous. So, $\pi_{Y} \circ h \circ j=\left(f \circ \pi_{X}\right)^{\prime} \circ j$. But, $\left(f \circ \pi_{X}\right)^{\prime} \circ j=f \circ \pi_{X}$. This shows that $\pi_{Y} \circ(h \circ j)=f \circ \pi_{X}$ and $f$ has a continuous $E$-lifting.

CoRollary 5.6. A function $f: X \rightarrow Y$ has a continuous E-lifting if

(a) $f$ is a weakly-continuous, closed irreducible surjection, or

(b) $f$ is weakly-continuous and open.

Proof. The proof follows from 3.4 and 3.5. 
If $X$ and $Y$ are spaces, then $X \oplus Y$ is used to denote the topological sum of $X$ and $Y$; the underlying set of $X \oplus Y$ is $X \times$ $\{1\} \cup Y \times\{2\}$. If $f: X \rightarrow Z$ and $g: Y \rightarrow Z$ are functions, then $f+g$ : $X \oplus Y \rightarrow Z$ is defined by $(f+g)(x, 1)=f(x)$ and $(f+g)(y, 2)=g(y)$. If $h: Y \rightarrow W$, then $f \oplus h: X \oplus Y \rightarrow Z \oplus W$ is defined by $(f \oplus h)(x, 1)=$ $(f(x), 1)$ and $(f \oplus h)(y, 2)=(h(y), 2)$. The next lemma is used in the proof of the characterization theorem for continuous $E$-liftings.

Lemma 5.7. Let $f: X \rightarrow Y$ be a function, $j: Y \rightarrow Y$ be the identity function, and $g=f+j: X+Y \rightarrow Y$. Then

(a) $f$ is $\eta$-continuous if and only if $g$ is $\eta$-continuous,

(b) $f$ is the composition of $\eta$-continuous functions if and only if $g$ is the composition of $\eta$-continuous functions, and

(c) $f$ has a continuous E-lifting if and only if $g$ has a continuous E-lifting.

Proof. The proof is long but straightforward and left to the reader.

It is important to note that the function $g$ in 5.7 is a surjection.

THEOREM 5.8. Let $f: X \rightarrow Y$ be a function. The following are equivalent:

(a) $f$ has a continuous E-lifting,

(b) $f$ is the composition of $\eta$-continuous functions, and

(c) $f$ is the composition of continuous functions and $\theta$-continuous, perfect, irreducible surjections.

Proof. By 5.5 and 5.6, (c) implies (a), and, by 3.9, (b) implies (c). To show (a) implies (b), suppose $f$ has a continuous $E$-lifting. By 5.7, we can assume that $f$ is onto. By 5.4, there is a Boolean algebra homomorphism $\varphi: R O(Y) \rightarrow R O(X)$ such that for $U \in R O(Y)$, $f^{-1}(U) \leqq \varphi(U) \leqq \operatorname{cl} \varphi(U) \leqq f^{-1}(\operatorname{cl} U)$. Let $X^{+}$denote the underlying set of $X$ plus the topology generated by the basis $\{\varphi(U): U \in R O(Y)\}$. Let $i^{+}: X \rightarrow X^{+}$be the identity function and $f^{+}: X^{+} \rightarrow Y$ be defined by $f^{+}(x)=f(x)$. For $A \subseteq X^{+}$, let $\mathrm{cl}^{+} A$ (resp. int ${ }^{+} A$ ) denote the closure of $A$ (resp. interior of $A$ ) in $X^{+}$. Clearly $i^{+}$is continuous. Next we will show that $f^{+}$is $\eta$-continuous. Let $U \in R O(Y)$. To show $\left(f^{+}\right)^{-1}(U) \subseteq$ int $^{+} \mathrm{cl}^{+}\left(f^{+}\right)^{-1}(U)$, it suffices to show $\varphi(U) \subseteq$ $\mathrm{cl}^{+}\left(f^{+}\right)^{-1}(U)$. Let $x \in \varphi(U)$ and let $\varphi(W)$ be a basic open set containing $x$ where $W \in R O(Y)$. Then $x \in \varphi(U) \cap \varphi(W)=\varphi(U \cap W)$. So, $U \cap W \neq \varnothing$ implying $\phi \neq\left(f^{+}\right)^{-1}(U \cap W) \subseteq\left(f^{+}\right)^{-1}(U) \cap \varphi(W)$. Thus, $\varphi(U) \subseteq \mathrm{cl}^{+}\left(f^{+}\right)^{-1}(U)$ and $\left(f^{+}\right)^{-1}(U) \subseteq \mathrm{int}^{+} \mathrm{cl}^{+}\left(f^{+}\right)^{-1}(U)$. Also this 
shows that $\mathrm{cl}^{+} \varphi(U)=\mathrm{cl}^{+}\left(f^{+}\right)^{-1}(U)$ and int $\mathrm{cl}^{+} \varphi(U)=$ int $^{+} \mathrm{cl}^{+} f^{-1}(U)$. Now, if $U, V R O(Y)$, then int $\mathrm{cl}^{+}\left(f^{+}\right)^{-1}(U \cap V)=\operatorname{int}^{+} \mathrm{cl}^{+} \varphi(U \cap V)=$ int $\mathrm{cl}^{+} \varphi(U) \cap$ int $^{+} \mathrm{cl}^{+} \varphi(V)=$ int $^{+} \mathrm{cl}^{+}\left(f^{+}\right)^{-1}(U) \cap$ int $^{+} \mathrm{cl}^{+}\left(f^{+}\right)^{-1}(V)$. This completes the proof that $f^{+}$is $\eta$-continuous. Since $f=f^{+} \circ i^{+}$, then (a) implies (b).

By 3.9 and the proof of 5.8, the composition of any finite number of $\eta$-continuous functions is the composition $f_{1} \circ f_{2} \circ f_{3} \circ f_{4}$ where $f_{2}$ and $f_{4}$ are continuous functions and $f_{1}$ and $f_{3}$ are $\theta$-continuous, closed bijections. However as shown by the next example, the composition of $\eta$-continuous functions need not be $\eta$-continuous.

EXAMPLE. Let $N$ denote the set of positive integers, and $Y=$ $\{(0,1)\} \cup\{(1 / n, 1 / m): n, m \in N\} \cup\{(1 / n, 0): n \in N\} \cup\{(1 / n,-1 / m): n, m \in N\}$. The topology for $Y \backslash\{(0,1)\}$ is the topology inherited from the plane; a basic open set of $(0,1)$ is $U_{p}=\{(0,1)\} \cup\{(1 / n,-1 / m): n>p, m \in N\}$ where $p \in N$. Let $Y_{1}=\operatorname{cl} U_{1}$ and $Y_{2}=Y \backslash U_{1}$. Let $X_{1}$ be the underlying set of $Y_{1}$ with $Y_{1} \backslash\{(0,1)\}$ discrete and a basic open set of $(0,1)$ is $V_{p}=\{(0,1)\} \cup\{(1 / n, 0): n \geqq p\}$ where $p \in N$. Let $X=X_{1} \oplus Y_{2}$. Let $j_{1}: Y_{1} \rightarrow Y_{1}$ and $j_{2}: Y_{2} \rightarrow Y_{2}$ be the identity functions and $g=$ $j_{1}+j_{2}: Y_{1} \oplus Y_{2} \rightarrow Y$. So, $g$ is continuous. Let $s:\left(Y_{1}\right)_{s} \oplus\left(Y_{2}\right)_{s} \rightarrow$ $Y_{1} \oplus Y_{2}$ be the identity function; since $\left(Y_{1} \oplus Y_{2}\right)_{s}=\left(Y_{1}\right)_{s} \oplus\left(Y_{2}\right)_{s}$, then $s$ is a $\theta$-continuous, perfect, irreducible bijection. The identity function $h: X \rightarrow\left(Y_{1}\right)_{s} \oplus\left(Y_{2}\right)_{s}$ is continuous. Let $f=g \circ s \circ h$. By 5.8, $f: X \rightarrow Y$ has a continuous $E$-lifting. Now, $U_{1}$ is a regular open set in $Y$ and bd $U_{1}=\{(1 / n, 0): n \in N\}$. Now, int $f^{-1}\left(\right.$ bd $\left.U_{1}\right)=\left\{(1 / n, 0) \in X_{1}\right.$ : $n \in N\}$ and $(0,1) \in \operatorname{cl} \operatorname{int} f^{-1}\left(\right.$ bd $\left.U_{1}\right)$. But $f(0,1) \notin$ bd $U_{1}$, and by $3.2, f$ is not $\eta$-continuous. It follows from 3.8 that $g \circ s$ is $\eta$-continuous since $\left(\left(Y_{1}\right)_{s} \oplus\left(Y_{2}\right)_{s}\right)^{*}=Y_{1} \oplus Y_{2}$. So, $f=(g \circ s) \circ h$ is the composition of two $\eta$-continuous surjections but is not $\eta$-continuous.

This paper is concluded with a necessary and sufficient condition for an $\eta$-continuous function to have a unique continuous $E$-lifting. This result parallels the corresponding result for $a$-liftings - see Proposition 2.5.

THEOREM 5.9. An $\eta$-continuous function has a unique continuous E-lifting if and only if it is a c-function.

Proof. The proof of the sufficiency is the same proof as for unique $a$-lifting. To prove the necessity, suppose $f: X \rightarrow Y$ is an $\eta$ continuous with a unique continuous $E$-lifting. By 3.9, $f=s \circ g \circ h$ where $g: X^{*} \rightarrow Y_{s}$ is continuous and $h: X \rightarrow X^{*}$ and $s: Y_{s} \rightarrow Y$ are $\theta$-continuous, perfect, irreducible bijections (and separating since one-to-one). For $U \in R O\left(X^{*}\right)$, int $h^{-1}($ bd $U)=\operatorname{int}\left(h^{-1}(\operatorname{cl} U) \backslash h^{-1}(U)\right)=$ int $h^{-1}(\operatorname{cl} U) \backslash \operatorname{cl} h^{-1}(U)$. By 3.5, int $h^{-1}(\operatorname{cl} U)=\operatorname{int} \operatorname{cl} h^{-1}(U)$. So, 
int $h^{-1}($ bd $U)=\varnothing$ and $h$ is $c$-function. Similarly, $s$ is $c$-function. By sufficiency portion of this theorem, $h$ and $s$ have unique continuous $E$-liftings $H$ and $S$, respectively. By 4.2, $H$ and $S$ are homeomorphisms. Assume $G_{1}$ and $G_{2}$ are direct continuous $a$-liftings of $g$. By 1.5, there are distinct continuous $E$-liftings $F_{1}$ and $F_{2}$ of $g$. Since $S$ and $H$ are homeomorphisms, $S \circ F_{1} \circ H$ and $S \circ F_{2} \circ H$ are distinct continuous $E$-liftings of $f$; this is a contradiction as $f$ has a unique continuous $E$-lifting. So, $g$ has a unique continuous $a$-lifting. By 2.5, $g$ is a $c$-function. Let $U \in R O(Y)$. Then $U$ is open in $Y_{s}$, and by 3.7 , bd $U=\mathrm{bd}_{s} U$. So, int $f^{-1}($ bd $U) \subseteq \operatorname{int}\left(f^{*}\right)^{-1}($ bd $U)=$ int* $g^{-1}\left(\mathrm{bd}_{s} U\right)=\varnothing$ implying $g$ is a $c$-function.

\section{REFERENCES}

1. W. W. Comfort and S. Negrepontis, The Theory of Ultrafilters, Springer, New York, 1974.

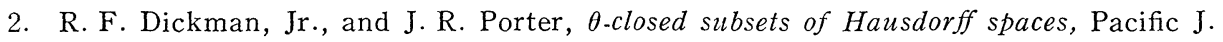
Math., 59 (1975), 407-415.

3. V. V. Fedorcuk, On H-closed extensions of $\theta$-proximity spaces, Math. USSR Sbornik, 18 (1972), 407-424.

4. L. Gillman and M. Jerison, Rings of Continuous Functions, Van Nostrand, New York, 1960.

5. H. Herrlich and C. E. Strecker, Category Theory, Allyn and Bacon, Boston, 1973.

6. T. Husain, Almost Continuous Mappings, Prace. Mat., 10 (1966), 1-7.

7. S. Iliadis and S. Fomin, The method of centered systems in the theory of topological spaces, Russian Math. Surveys, 21 (1966), 37-62.

8. C.-T. Liu, Absolutely closed spaces, Trans, Amer. Math. Soc., 130 (1968), 86-104.

9. J. Mioduszewski and L. Rudolf, H-closed extremally disconnected Hausdorff spaces, Dissert. Math., 66 (1969), 1-55.

10. J. R. Porter and J. D. Thomas, On H-closed and minimal Hausdorff spaces, Trans. Amer. Math. Soc., 138 (1969), 159-170.

11. L. B. Sapiro, On absolutes of topological spaces and continuous mappings, Soviet Math. Dokl., 17 (1976), 147-151.

12. D. P. Strauss, Extremally disconnected spaces, Proc. Amer, Math. Soc., 18 (1967), 305-309.

13. V. M. Ul'janov, On compactifications satisfying the first axiom of countability and absolutes, Math. USSR Sbornik, 27 (1975), 199-226.

14. R. C. Walker, The Stone-Cech Compactification, Springer, New York, 1974.

15. R. G. Woods, A survey of absolutes of topological spaces, Topological Structures II, Math. Centre Tracts 116 (1979), 323-362.

Received February 8, 1980 and in revised form May 29, 1980. The research of the second author was partially supported by the University of Kansas General Research Fund.

Virginia Polytechnic Institute and State University

BlacksBURG, VA 24061

UNIVERSITY OF KANSAS

LAWRENCE, KS 66045

AND

University of OkLahoma

Norman, OK 73069 



\section{PACIFIC JOURNAL OF MATHEMATICS}

\section{EDITORS}

DONALD BABBITT (Managing Editor)

University of California

Los Angeles, CA 90024

Hugo RossI

University of Utah

Salt Lake City, UT 84112

C. C. MOORE and ANDREW OGG

University of California

Berkeley, CA 94720
J. DugundjI

Department of Mathematics

University of Southern California

Los Angeles, CA 90007

R. FINN and J. MILGRAM

Stanford University

Stanford, CA 94305

\section{ASSOCIATE EDITORS}
R. ARENS
E. F. BECKENBACH
B. H. NeUmanN
F. WOLF
K. YosHIDA

\section{SUPPORTING INSTITUTIONS}

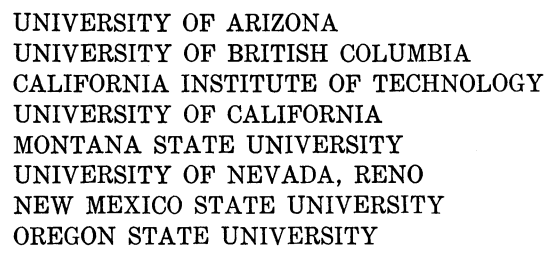

UNIVERSITY OF ARIZONA

UNIVERSITY OF BRITISH COLUMBIA CALIFORNIA INSTITUTE OF TECHNOLOGY

UNIVERSITY OF CALIFORNIA

MONTANA STATE UNIVERSITY

UNIVERSITY OF NEVADA, RENO

NEW MEXICO STATE UNIVERSITY OREGON STATE UNIVERSITY

\author{
UNIVERSITY OF OREGON \\ UNIVERSITY OF SOUTHERN CALIFORNIA \\ STANFORD UNIVERSITY \\ UNIVERSITY OF HAWAII \\ UNIVERSITY OF TOKYO \\ UNIVERSITY OF UTAH \\ WASHINGTON STATE UNIVERSITY \\ UNIVERSITY OF WASHINGTON
}

The Supporting Institutions listed above contribute to the cost of publication of this Journal, but they are not owners or publishers and have no responsibility for its content or policies.

Mathematical papers intended for publication in the Pacific Journal of Mathematics should be in typed form or offset-reproduced, (not dittoed), double spaced with large margins. Please do not use built up fractions in the text of the manuscript. However, you may use them in the displayed equations. Underline Greek letters in red, German in green, and script in blue. The first paragraph or two must be capable of being used separately as a synopsis of the entire paper. Please propose a heading for the odd numbered pages of less than 35 characters. Manuscripts, in triplicate, may be sent to any one of the editors. Please classify according to the scheme of Math. Reviews, Index to Vol. 39. Supply name and address of author to whom proofs should be sent. All other communications should be addressed to the managing editor, or Elaine Barth, University of California, Los Angeles, California, 90024.

50 reprints to each author are provided free for each article, only if page charges have been substantially paid. Additional copies may be obtained at cost in multiples of 50 .

The Pacific Journal of Mathematics is issued monthly as of January 1966. Regular subscription rate: $\$ 102.00$ a year (6 Vols., 12 issues). Special rate: $\$ 51.00$ a year to individual members of supporting institutions.

Subscriptions, orders for numbers issued in the last three calendar years, and changes of address shoud be sent to Pacific Journal of Mathematics, P.O. Box 969, Carmel Valley, CA 93924, U.S.A. Old back numbers obtainable from Kraus Per!odicals Co., Route 100, Millwood, NY 10546.

\section{PUBLISHED BY PACIFIC JOURNAL OF MATHEMATICS, A NON-PROFIT CORPORATION}

Printed at Kokusai Bunken Insatsusha (International Academic Printing Co., Ltd.). 8-8, 3-chome, Takadanobaba, Shinjuku-ku, Tokyo 160, Japan.

Copyright (C) 1981 by Pacific Jounal of Mathematics Manufactured and first issued in Japan 


\section{Pacific Journal of Mathematics}

Vol. 94, No. $2 \quad$ June, 1981

Thomas E. Armstrong and William David Sudderth, Nearly strategic

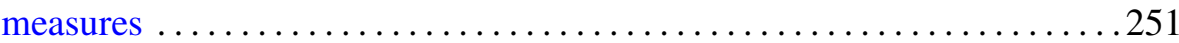

John J. Buoni, Artatrana Dash and Bhushan L. Wadhwa, Joint Browder

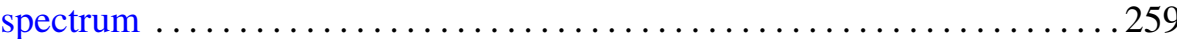

Jack Paul Diamond, Hypergeometric series with a $p$-adic variable . .......265

Raymond Frank Dickman, Jack Ray Porter and Leonard Rubin,

Completely regular absolutes and projective objects ............ 277

James Kenneth Finch, On the local spectrum and the adjoint ........... 297

Benno Fuchssteiner, An abstract disintegration theorem ............ 303

Leon Gerber, The volume cut off a simplex by a half-space $\ldots \ldots \ldots \ldots 311$

Irving Leonard Glicksberg, An application of Wermer's subharmonicity

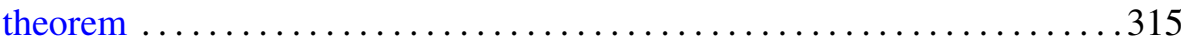

William Goldman, Two examples of affine manifolds ............... 327

Yukio Hirashita, On the Weierstrass points on open Riemann surfaces .....331

Darrell Conley Kent, A note on regular Cauchy spaces ............. 333

Abel Klein and Lawrence J. Landau, Periodic Gaussian

Osterwalder-Schrader positive processes and the two-sided Markov

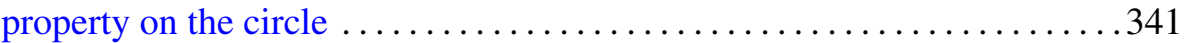

Brenda MacGibbon, $\mathscr{K}$-Borelian embeddings and images of Hausdorff

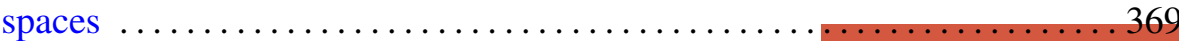

John R. Myers, Homology 3-spheres which admit no PL involutions . . . . . 379

Boon-Hua Ong, Invariant subspace lattices for a class of operators . . . . . 385

Chull Park, Representations of Gaussian processes by Wiener processes . . . 407

Lesley Millman Sibner and Robert Jules Sibner, A sub-elliptic estimate

for a class of invariantly defined elliptic systems $\ldots \ldots \ldots \ldots \ldots \ldots .417$

Justin R. Smith, Complements of codimension-two submanifolds. III.

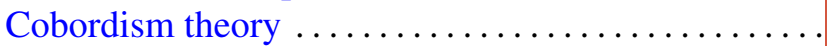

William Albert Roderick Weiss, Small Dowker spaces

David J. Winter, Cartan subalgebras of a Lie algebra and its ideals. II ... 